\section{Posterior vitreous detachment following cataract surgery}

D Hilford, M Hilford, A Mathew and

PJ Polkinghorne

\section{Introduction}

Pseudophakic retinal detachment (PRD) is a well-recognised complication of cataract surgery and is reported to occur in approximately $1 \%$ of eyes undergoing cataract surgery using phacoemulsification techniques. ${ }^{1-3}$ The risk is greater in eyes where the axial length exceeds $25 \mathrm{~mm}$ and in eyes where surgery is complicated by vitreous loss. ${ }^{1,4}$ Interestingly the effect of performing a neodymium: yttrium garnet $(\mathrm{Nd}: \mathrm{YAG})$ capsulotomy in the perioperative period is not always reported as increasing the risk of PRD. ${ }^{5}$

In our population, age has been shown to impact the incidence of PRD, and the importance of age as a risk factor has been confirmed in two other recent reports. ${ }^{1,6}$ In our own series, we found the risk of PRD for individuals undergoing cataract surgery by phacoemulsification when aged 60 years or less approximated $6 \% .^{7}$ This contrasted with a risk of $0.6 \%$ for those individuals who had cataract surgery at the age of 70 years or above.

To date, no adequate explanation has been substantiated as to why the younger patient undergoing cataract surgery is at greater risk of PRD. ${ }^{8,9}$ It follows, however, any age-related effect is likely to be more prevalent in a cohort of younger patients undergoing cataract surgery, and the rationale for undertaking this study was an attempt to identify any such risk factors.

Patients and methods

We identified, from the surgical logbooks of a private institution, all those patients undergoing planned cataract surgery by phacoemulsification aged between 50 and 60 years during 1999-2002. All surgeries were performed by consultant surgeons in a facility, which is accredited by Quality Health New Zealand. Ethics approval was obtained from the
Keywords: posterior vitreous detachment; phacoemulsification; cataract; surgery Eye (2009) 23, 1388-1392; doi:10.1038/eye.2008.273; published online 5 September 2008

\footnotetext{
Received: 23 June 2008 Accepted in revised form: 12 August 2008 Published online: 5 September 2008

Department of of Auckland, Auckland,

Correspondence:

Department of

Ophthalmology, PB 92019,

Auckland,

Tel: + 649529 2480;

Fax: + 6495292481

E-mail: Philip@

(
} 
Human Research Ethics Committee at the Royal Australian and New Zealand College of Ophthalmologists.

Patients who meet the inclusion criteria were recalled for an ophthalmic examination during the first half of 2007. We collated age and sex of the patient, and recorded any relevant ophthalmic history. Specific enquiry was directed to establish the existence of previous posterior segment pathology.

The availability of the preoperative biometry enabled us to record the anterior chamber depth as well as axial length. More than 95\% of this data were sourced using the IOL master (Zeiss, Germany). The date of the cataract surgery as well as any postoperative complication including retinal tear or PRD were noted. The presence or absence of a capsulotomy was determined during the ophthalmic assessment, which also included a dilated retinal examination of both eyes. The presence of lattice degeneration was noted. This was defined as a lesion of the peripheral retina, orientated in a circumferential or radial pattern, with a lattice pattern or arborising lines in the lesion with adherent, condensed vitreoretinal attachments at the margins.

The diagnosis of a posterior vitreous detachment (PVD) was established by the existence of a Weiss ring. If a Weiss ring was absent, then a real-time ultrasound was performed using the OTI-2000 (Ophthalmic Technologies Inc., Canada). This machine is fitted with a $10 \mathrm{MHz}$ probe. For this study, the manufacturers advised that the gain should be set to $75-80 \mathrm{~dB}$ to ensure the maximum reliability of detecting the posterior hyaloid. The vitreous was considered detached, if there was a characteristic movement of the posterior vitreous with ocular movements as well as an echolucent interval between the retina and posterior hyaloid.

Patients that had undergone bilateral cataract surgery on both eyes included in the study, providing the information that the surgeries were performed during the sixth decade of life. If one surgery was performed outside this 10-year window, that eye was excluded. Patients who were deceased at the time of recall were also excluded from data analysis, as were those eyes that had undergone vitrectomy surgery before recall.

\section{Data analysis}

Statistical analyses were performed in conjunction with a professional biomedical statistician. The data were initially entered on a Microsoft Excel database and then subsequently exported to statistical software for analysis. Statistical analysis was performed in SPSS Version 12 for Windows (Chicago, IL, USA) and SAS 9.1(SAS Institute Inc.). Basic descriptive statistics were calculated on all data gathered and are reported as mean \pm SD or $n(\%)$ as appropriate. Likelihood ratio, $\chi^{2}$-tests, and Fisher's exact tests were applied, as appropriate for testing associations between categorical variables. Correlations between continuous variables were examined by calculating either Pearson's or Spearman's correlation coefficient $(r)$.

The categorical variables tested for associations included the male and female sex, presence or absence of an Nd:YAG capsulotomy, axial lengths less and greater or equal to $25 \mathrm{~mm}$, and finally, the presence or absence of vitreous floaters.

Univariate logistic regressions were applied to those parameters that might be associated with the development of a PVD in the perioperative period. These parameters tested included age, sex, history of Nd:YAG capsulotomy, axial length; anterior chamber depth, as well as the preoperative refraction.

Risk factors with $P$-values $<0.15$ were further evaluated with multiple logistic regression analyses. Plots of sensitivity and specificity were determined with the receiver operating characteristic curves (ROC) and the area under the curve used as a criterion of prediction accuracy. An additional analysis was performed by applying the generalised estimated equation (GEE) to model binomial outcomes, adjusting for the potential correlation of paired eyes for each patient. All tests were two-tailed and a $P$-value of $\leqslant 0.05$ was considered statistically significant.

\section{Results}

In all, 149 consecutive patients were identified as being eligible for recall based on the entry criteria. Of these, four patients were deceased and were subsequently excluded. A further 12 patients who had undergone vitrectomy surgery in the study eye were also excluded. Twenty-two patients declined or were unavailable to attend a recall examination limiting the study to 111 patients $(83 \%)$ from the original cohort. This enabled 170 eyes comprising of 24 phakic and 146 pseudophakic eyes to be included in the analysis. The median interval between surgery and recall was 77 months (range 23-96). The mean age of the patients undergoing surgery was 55 years (range $50-59)$ with $69 \%(n=77)$ of female participants (Table 1).

One eye in this study had a history of retinal reattachment surgery before undergoing uncomplicated cataract surgery. Another two eyes, although still phakic, had also had been treated for retinal breaks. None of these eyes developed further retinal sequelae. Six eyes before the recall examination did develop a PRD equating to a detachment rate of $4 \%$. All were successfully treated. Twelve pseudophakic eyes were noted at recall to have lattice degeneration. No eye with 
lattice degeneration developed a PRD until the time of recall.

The incidence of PVD in the eyes still phakic at the time of the review was $20.8 \%$. $(n=5)$ A Weiss ring was identified in three of these eyes with the remaining two eyes being diagnosed on B-scan ultrasonography. A PVD was present in $96(50.8 \%)$ of the pseudophakic eyes and this difference was statistically significant $(P<0.0001)$.

Those pseudophakic eyes with an axial length of $\geqslant 25 \mathrm{~mm}$ were also more likely to have a PVD as compared to those eyes with an axial length of $<25 \mathrm{~mm}$. Odds ratio $4.7,(1.6,14.4) P=0.006$.

Thirty-six eyes had undergone an Nd:YAG capsulotomy by the time of the recall examination, including 10 of 12 eyes with lattice degeneration. Twentyfive PVD were documented to have occurred in these eyes (69\%). This contrasted with 71 PVD in the remaining 110 pseudophakic eyes with intact posterior capsules. No

Table 1 Characteristics of patients enrolled in the study

Demography of patients

\begin{tabular}{ll}
\hline Number of patients enrolled in the study & 133 \\
Number of patients examined (\%) & $111(83 \%)$ \\
Number of pseudophakic eyes & 146 \\
Number of phakic eyes & 24 \\
Median age of patients (interquartile) & $56(53,58)$ \\
Male patients (\%) & $34(30 \%)$ \\
Median follow-up in months (min, max) & $77(15,147)$
\end{tabular}

Ophthalmic findings

\begin{tabular}{ll}
\hline $\begin{array}{l}\text { AC depth mean (SD) } \\
\text { Number of eyes with an axial length } \\
\text { of } \geqslant 25 \mathrm{~mm}(\%)\end{array}$ & $3.3(0.4)$ \\
Number of eyes with previous retinal tear & $13(8 \%)$ \\
Number of eyes with an Nd:YAG capsulotomy & $40(27 \%)^{\ddagger}$ \\
& \\
Patient outcomes & 0 \\
\hline $\begin{array}{l}\text { Eyes that developed isolated retinal tear } \\
\text { Eyes that developed an RRD }\end{array}$ & $4(2 \%)^{\ddagger}$ \\
Eyes with a PVD after cataract surgery & $96(50.8 \%)^{\ddagger}$
\end{tabular}

*All proportions divided by the total number of patients enrolled in the study except those proportions applicable to those pseudophakic eyes were denoted by symbol ${ }^{\ddagger}$. statistical difference was, therefore, observed in the incidence of PVD between those eyes with and without an Nd:YAG capsulotomy. $(P=0.59)$ We did not find any correlation between PVD and gender, age or preoperative refraction (Table 2 ).

However, univariate analysis did identify an inverse relationship between PVD and anterior chamber depth. The risk of developing a PVD being 3.3 times (95\% CI 1.4, $11.1 ; P=0.009)$ greater for every $0.1-\mathrm{mm}$ reduction in anterior chamber depth.

Multivariate logistic regression was used to re-examine those characteristics identified in the univariate analysis where the $P<0.15$. This stepwise selection confirmed the relevance of both anterior chamber depth and axial length and showed the area under the curve increased from 0.61 to 0.70 . A generalised estimate equation was used to repeat the calculation and a similar outcome was observed (Table 3).

We enquired, at the time of recall, whether the patient was currently aware of floaters, in either eye or had been in the past. Fifty-four eyes were reported to contain floaters, but of these, only 23 had a documented PVD. For those patients with a Weiss ring $(n=51)$, the reliability was greater with 30 eyes reported to contain floaters. This difference was statistically significant $(P<0.001)$. On the other hand, floaters were absent in 21 eyes with a Weiss ring. For eyes that were pseudophakic, the presence of floaters was not helpful in determining the presence of a PVD (Table 4).

\section{Discussion}

In this study, we have documented that PVD occurs more frequently in aged matched eyes that have undergone

Table 3 Multivariate analysis of anterior chamber depth and axial length and posterior vitreous detachment

\begin{tabular}{llll}
\hline Odds ratio & P-value & \multicolumn{1}{c}{$\begin{array}{c}\text { Direction of } \\
\text { the risk factor }\end{array}$} & AUC \\
\hline $0.2(0.05,0.51)$ & 0.002 & $\begin{array}{l}\text { Negative, the shorter, the riskier } \\
\text { Postive, the longer, the riskier }\end{array}$ & 0.70 \\
\hline $.4(2.0,20.8)$ & 0.002 & Pat
\end{tabular}

Table 2 Summary of relationship variables and presence of postvitreous detachment in pseudophakic eyes

\begin{tabular}{|c|c|c|c|}
\hline Risk factors & Odds ratio & P-value & $\begin{array}{c}\text { Area under receiver } \\
\text { operator characteristics curve }\end{array}$ \\
\hline Age & $1.0(0.9,1.1)$ & 0.94 & \\
\hline Gender & $0.9(0.5,1.8)$ & 0.83 & \\
\hline Preoperative refraction & $0.9(0.80,1.0)$ & 0.08 & \\
\hline Nd;YAG capsulotomy & $0.8(0.4,1.8)$ & 0.59 & \\
\hline Anterior chamber depth & $0.3(0.09,0.71)$ & 0.009 & 0.61 \\
\hline Axial length & $4.7(1.6,14.4)$ & 0.006 & 0.61 \\
\hline
\end{tabular}


Table 4 Reliability of floaters as an indicator of the presence of a posterior vitreous detachment in phakic and pseudophakic eyes

\begin{tabular}{|c|c|c|c|}
\hline \multicolumn{4}{|c|}{ Phakic eyes $(\mathrm{n}=24)$} \\
\hline \multicolumn{3}{|c|}{ Posterior vitreous detachment $(\mathrm{n}=5)$} & \multirow[t]{2}{*}{ Attached vitreous $(\mathrm{n}=19)$} \\
\hline & Weiss ring (present) $(\mathrm{n}=3)$ & Weiss ring (absent) $(\mathrm{n}=2)$ & \\
\hline Symptoms & 1 & 2 & 1 \\
\hline Asymptomatic & 2 & 0 & 18 \\
\hline \multicolumn{4}{|c|}{ Pseudophakic eyes $(\mathrm{n}=146)$} \\
\hline \multicolumn{3}{|c|}{ Posterior vitreous detachment $(\mathrm{n}=96)$} & \multirow[t]{2}{*}{ Attached vitreous $(\mathrm{n}=50)$} \\
\hline & Weiss ring (present) $(\mathrm{n}=48)$ & Weiss ring (absent) $(\mathrm{n}=48)$ & \\
\hline Symptoms & 29 & 6 & 16 \\
\hline Asymptomatic & 19 & 42 & 34 \\
\hline
\end{tabular}

cataract surgery by phacoemulsification as compared to phakic eyes. Furthermore, a PVD is more common in pseudophakic eyes where the axial length is equal or exceeds $25 \mathrm{~mm}$. We did not find that the presence of an Nd:YAG capsulotomy increased the likelihood of a PVD.

We limited this study to patients undergoing cataract surgery in their sixth decade, as this age group appears to have a greater risk of PRD. In our own population, we have determined this risk approximates $6 \%$, whereas for patients above 70 years, the risk is $0.6 \% .^{7}$ In this study with a median follow-up of just 77 months, the incidence of PRD was $4 \%$ which is in keeping with our previous study, which had a longer follow-up interval.

The relationship between PVD and rhegmatogenous retinal detachment is well established, although it is not clear why in some eyes the development of a PVD precipitates a retinal detachment, whereas in apparently similar eyes, the same event is uncomplicated. ${ }^{10}$ Indeed, our inability to identify eyes at greater risk has lead to an advice that all eyes presenting with symptoms indicative of a PVD should have a retinal examination. ${ }^{11}$

Unfortunately, symptoms are not always a reliable indicator of risk, and in this study, the concordance between PVD and symptoms in the pseudophakic eye was $<50 \%$. Symptoms of floaters were more reliable in this subset, if the PVD was associated with a Weiss ring.

Ripandelli et $a l^{9}$ also have reviewed the incidence of PVD following cataract surgery and determined the risk of PRD. Their selection criteria included patients aged 50-72 years, although their survey was restricted to eyes that were emmetropic. They found the incidence of PVD following cataract surgery was not in fact age related, although PRD did occur more frequently in the younger age group. These authors also found a high incidence of lattice degeneration in the younger age group and linked this, in presence of a PVD, to PRD. In our study, the incidence of lattice degeneration was approximately $8 \%$, which was similar (10.4\%) to that documented by Ripandelli et al. ${ }^{9}$ However, unlike Ripandelli et al, ${ }^{9}$ none of the eyes with lattice degeneration in our series developed PRD; this is, in spite of all the eyes with lattice degeneration in our series, having the axial length $>25 \mathrm{~mm}$. This discrepancy may ultimately be resolved with a longer follow-up interval, but at present, our data would not support tha fact that the contention prophylactic treatment to lattice degeneration would prevent PRD.

The link between anterior chamber depth and risk of PRD has also been explored by Bhagwanien et al ${ }^{4}$ who found a deeper anterior chamber correlated with PRD. In that retrospective study, PVD was not directly assessed, although the authors postulated that the association of anterior chamber depth might be related to an increased risk of PVD. The rationale given for that statement being a deeper anterior chamber facilitates increased the transmission of vibrations to the anterior hyaloid during cataract surgery. In our study, we did not observe an increased risk of PVD with anterior chamber depth; indeed, we found the opposite, namely the risk of PVD was inversely related to anterior chamber depth. As to the biological plausibility of our finding, it may be the altered profile of an intraocular lens as compared to a crystalline lens in an eye with a shallower anterior chamber may permit greater mobility of the anterior hyaloid, increasing the risk of PVD. A similar mechanism may account for the higher PVD incidence in eyes with an axial length of $>25 \mathrm{~mm}$, and indeed may explain why both axial lengths $>25 \mathrm{~mm}$ and shallower anterior chamber depth, when analysed together, are more strongly associated with a PVD than when they are considered separately.

In summary, this study has explored a group of patients known to be at higher risk of PRD and set out to determine whether that risk could be linked to the induction of a PVD. We have not yet proven that link, but 
have demonstrated cataract surgery performed in the sixth decade of life is associated with an increased rate of PVD, and that rate is further modified by both axial length and a shallow anterior chamber depth.

\section{References}

1 Tuft SJ, Minassian D, Sullivan P. Risk factors for retinal detachment after cataract surgery: a case-control study. Ophthalmology 2006; 113(4): 650-656.

2 Lois N, Wong D. Pseudophakic retinal detachment. Surv Ophthalmol 2003; 48(5): 467-487.

3 Sheu SJ, Ger LP, Chen JF. Male sex as a risk factor for pseudophakic retinal detachment after cataract extraction in taiwanese adults. Ophthalmology 2007; 114(10): 1898-1903.

4 Bhagwandien AC, Cheng YY, Wolfs RC, van Meurs JC, Luyten GP. Relationship between retinal detachment and biometry in 4262 cataractous eyes. Ophthalmology 2006; 113: 643-649.

5 Olsen G, Olson RJ. Update on a long-term, prospective study of capsulotomy and retinal detachment rates after cataract surgery. J Cataract Refract Surg 2000; 26: 1017-1021.
6 Erie JC, Raecker ME, Baratz KH, Schleck CD, Robertson DM. Risk of retinal detachment after cataract extraction, 1980-2004: a population-based study. Trans Am Ophthalmol Soc 2006; 104: 167-175.

7 Russell M, Gaskin B, Russell D, Polkinghorne PJ. Pseudophakic retinal detachment after phacoemulsification cataract surgery: Ten-year retrospective review. J Cataract Refract Surg 2006; 32: 442-445.

8 Ripandelli G, Scassa C, Parisi V, Gazzaniga D, D’Amico DJ, Stirpe M. Cataract surgery as a risk factor for retinal detachment in very highly myopic eyes. Ophthalmology 2003; 110: 2355-2361.

9 Ripandelli G, Coppe AM, Parisi V, Olzi D, Scassa C, Chiaravalloti A et al. Posterior vitreous detachment and retinal detachment after cataract surgery. Ophthalmology 2007; 114: 692-697.

10 Byer NE. Natural history of posterior vitreous detachment with early management as the premier line of defense against retinal detachment. Ophthalmology 1994; 101: 1503-1513; discussion 13-4.

11 Ophthalmology AAO. Management of Posterior Vitreous Detachment, Retinal Breaks, and Lattice Degeneration. Preferred practice Pattern. American Academy of Ophthalmology: San Francisco, 2003 v.2007. 